\title{
Free Wall Myocardial Rupture and Ventricular Septal Defect in an Ambulatory Patient
}

\author{
Waqas Memon ${ }^{1}$, Muhammed Aamir ${ }^{2}$, Areeka Memon ${ }^{3}$, Maha Mikhail ${ }^{4}$ \\ 1. Internal Medicine/Nephrology, Virginia Commonwealth University, Richmond, USA 2. Internal Medicine, Carle \\ Foundation Hospital, Urbana, USA 3. Osteopathic Medicine, Edward Via College of Osteopathic Medicine, Blacksburg, \\ USA 4. Cardiology, Carle Foundation Hospital, Urbana, USA
}

Corresponding author: Waqas Memon, waqasm03@hotmail.com

\begin{abstract}
Myocardial rupture is a rare complication of acute myocardial infarction (MI), usually presenting with chest pain. The most common site of rupture is the anterior wall. Myocardial rupture presents similar to cardiac tamponade, most frequently as cardiogenic shock. Many clinical conditions, however, present similarly. The differential diagnosis should include myocardial rupture if clinical suspicion is high. This report describes a 77-year-old man with a medical history putting him at significant risk for coronary artery disease status, including a coronary artery bypass graft, chronic kidney disease stage 3 , and hyperlipidemia. He presented at the ED for worsening shortness of breath and feeling unwell. Transthoracic echocardiography revealed an anterior, anterolateral akinesis, a ventricular septal defect, and free wall rupture. Myocardial rupture is an acute cardiac emergency; a high level of clinical suspicion may help in detecting this rare manifestation of acute MI.
\end{abstract}

Categories: Cardiology, Emergency Medicine, Internal Medicine

Keywords: myocardial rupture, cardiology, critical care, rare presentation, myocardial infarction

\section{Introduction}

Myocardial rupture is a rare and serious complication of acute myocardial infarction (MI), usually presenting as an acute cardiac emergency. The most common symptom on presentation is chest pain, and the most common site of rupture is the anterior wall [1-2]. The overall incidence of free wall rupture is declining, from $>4 \%$ in 1977-1982 to <2\% in 2001-2006, a decrease attributed to the advances in reperfusion therapies and better clinical awareness of blood pressure goals [3].

\section{Case Presentation}

A 77-year-old man with a past medical history putting him at significant risk for coronary artery disease, including a coronary artery bypass graft, chronic kidney disease stage 3 , and hyperlipidemia presented at the ED for worsening shortness of breath and feeling unwell. An initial examination showed that the patient was hypotensive and tachycardic, with $98 \%$ oxygen saturation on room air. He was positive for diffuse crackles over the right and left hemithorax without any focal neurological findings. Cardiac examination showed that he was positive for jugular vein distention and trace lower extremity edema (Table 1 ).

Received 05/12/2020 Review ended 05/26/2020 Published 06/06/2020

\section{๑) Copyright 2020}

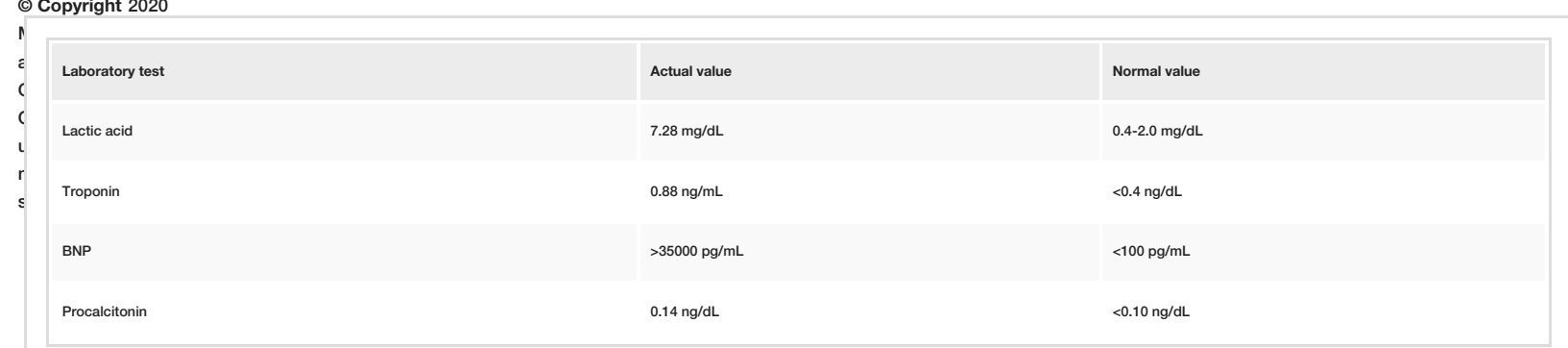

\section{TABLE 1: Laboratory tests.}

BNP, brain natriuretic peptide

Because of high clinical suspicion of impending shock, the patient was started on broad-spectrum antibiotics and norepinephrine for vasopressor support. He became hypoxic and required bilevel positive airway pressure and vasopressor support. Transthoracic echocardiography revealed anterior, anterolateral akinesis, a ventricular septal defect, and free wall rupture, findings confirmed by both echo/contrast echo and transesophageal echocardiography (TEE) showing contrast in the pericardial sac (Videos 1-2). 


\section{Cureus}

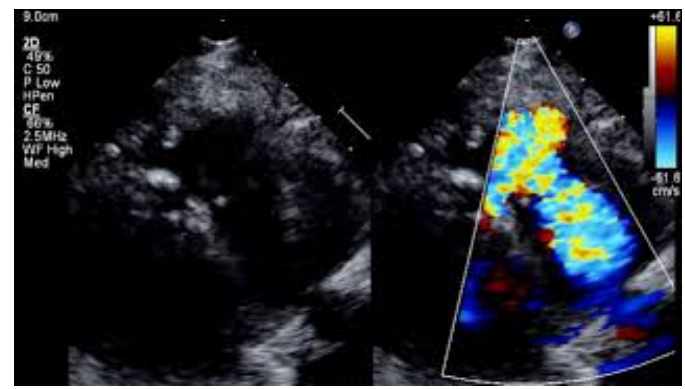

VIDEO 1: Transthoracic echocardiogram depicting severely reduced LV systolic function with regional wall motion abnormalities. The anterior wall was severely hypokinetic, and the anterolateral wall was akinetic with scarring and wall thinning. A contained LV free wall rupture at the mid-cavity level was noted. Additionally, a large ventricular septal defect was seen in the mid and apical segment of the myocardium. The right ventricle was dilated and severely hypokinetic. Biatrial enlargement was present.

LV, left ventricular

View video here: https://youtu.be/brTDSJiN7_Y

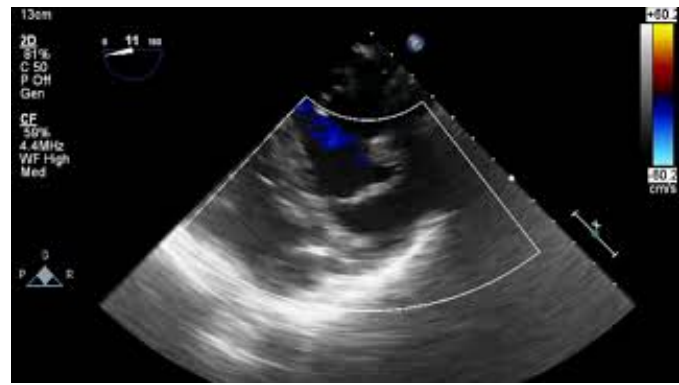

VIDEO 2: Transthoracic echocardiogram at the mid esophageal level showing the short axis of the LV with confirmation of free wall rupture of the anterolateral wall of the LV at the mid cavity with ventricular septal defect.

LV, left ventricle

View video here: https://youtu.be/2gM3WoGy8Vu

The patient was switched to dobutamine for inotropic support, and emergency cardiovascular surgery was considered. The patient continued to do poorly, requiring increased vasopressor and ventilator support. After consultation with his family, the patient was made comfortable and died.

\section{Discussion}

Myocardial rupture presents similar to cardiac tamponade, most frequently as cardiogenic shock resulting in sudden cardiac death [2-4]. Without proper imaging and/or an autopsy, death in these patients can be attributed to fatal arrhythmia, heart block, or massive pulmonary embolism [5]. Determining proper treatment is difficult because many clinical conditions share similar presentations. Thus, the differential diagnosis of these patients should include myocardial rupture if clinical suspicion is high.

Myocardial rupture can be diagnosed by standard TEE, although contrast-enhanced echocardiography can also be helpful. Cardiac MRI can also help confirm an impending myocardial rupture. Management depends on early recognition of myocardial rupture, with immediate medical therapy including the administration of intravenous fluids, inotropes agents, and vasopressors to maintain patient hemodynamics and prevent or reduce shock [6]. Cardiothoracic surgery is the definitive treatment [7-9]. 


\section{Conclusions}

Early diagnosis of myocardial rupture early is extremely important in preventing catastrophic outcomes. Myocardial rupture should be included in the differential diagnosis of patients presenting with acute MI. Early recognition, stabilization, and emergency surgical intervention can improve patient survival.

\section{Additional Information}

\section{Disclosures}

Human subjects: Consent was obtained by all participants in this study. Conflicts of interest: In compliance with the ICMJE uniform disclosure form, all authors declare the following: Payment/services info: All authors have declared that no financial support was received from any organization for the submitted work. Financial relationships: All authors have declared that they have no financial relationships at present or within the previous three years with any organizations that might have an interest in the submitted work. Other relationships: All authors have declared that there are no other relationships or activities that could appear to have influenced the submitted work.

\section{References}

1. Hutchins KD, Skurnick J, Lavenhar M, Natarajan GA: Cardiac rupture in acute myocardial infarction: a reassessment. Am J Forensic Med Pathol. 2002, 23:78-82. 10.1097/00000433-200203000-00017

2. Becker RC, Hochman JS, Cannon CP, Spencer FA, Ball SP, Rizzo MJ, Antman EM: Fatal cardiac rupture among patients treated with thrombolytic agents and adjunctive thrombin antagonists: observations from the Thrombolysis and Thrombin Inhibition in Myocardial Infarction 9 Study. J Am Coll Cardiol. 1999, 33:479-487. 10.1016/S0735-1097(98)00582-8

3. Honda S, Asaumi Y, Yamane T, et al.: Trends in the clinical and pathological characteristics of cardiac rupture in patients with acute myocardial infarction over 35 years. J Am Heart Assoc. 2014, 3:000984. 10.1161/JAHA.114.000984

4. Pouleur AC, Barkoudah E, Uno H, et al.: Pathogenesis of sudden unexpected death in a clinical trial of patients with myocardial infarction and left ventricular dysfunction, heart failure, or both. Circulation. 2010, 122:597-602. 10.1161/CIRCULATIONAHA.110.940619

5. Hayes SN, Kim ESH, Saw J, et al.: Spontaneous coronary artery dissection: current state of the science: a scientific statement from the American Heart Association. Circulation. 2018, 137:523-557. 10.1161/CIR.0000000000000564

6. DeFilippis AP, Chapman AR, Mills NL, de Lemos J, Arbab-Zadeh A, Newby LK, Morrow DA: Assessment and treatment of patients with type 2 myocardial infarction and acute nonischemic myocardial injury. Circulation. 2019, 140:1661-1678. 10.1161/CIRCULATIONAHA.119.040631

7. López-Sendón J, González A, López de Sá E, et al.: Diagnosis of subacute ventricular wall rupture after acute myocardial infarction: sensitivity and specificity of clinical, hemodynamic and echocardiographic criteria. J Am Coll Cardiol. 1992, 19:1145-1153. 10.1016/0735-1097(92)90315-E

8. Amir O, Smith R, Nishikawa A, Gregoric ID, Smart FW: Left ventricular free wall rupture in acute myocardial infarction: a case report and literature review. Tex Heart Inst J. 2005, 32:424-426.

9. Rogowski S, Maeder MT, Weilenmann D, et al.: Spontaneous coronary artery dissection: angiographic follow-up and long-term clinical outcome in a predominantly medically treated population. Catheter Cardiovasc Interv. 2017, 89:59-68. 10.1002/ccd.26383 